\title{
Mi visión ante el paciente hipertenso
}

\author{
My personal outlook of a hypertensive patient \\ Dagnóvar Aristizábal-Ocampo* \\ Dirección Científica, Centro Clínico y de Investigación SICOR, Medellín, Colombia
}

A comienzos de este siglo, en el año 2005, tuve la oportunidad de escribir un artículo editorial para nuestra revista ${ }^{1}$, en el cual indiqué los grandes cambios que nos esperaban en hipertensión arterial en el siglo XXI. En particular hice referencia a la promesa no cumplida de la genética para darnos respuestas al origen de la hipertensión esencial y cómo la alta carga de factores ambientales, particularmente el sobrepeso y el estrés psicosocial, generaría una mayor prevalencia de hipertensión esencial en nuestro país y en Latinoamérica. El reloj ha seguido su marcha y gran parte de lo esperado se está observando actualmente. En este editorial presentaré brevemente los avances logrados en los últimos tres lustros fruto de una activa investigación, y también mencionaré lo que nos falta por mejorar en términos diagnósticos y terapéuticos en la hipertensión llamada esencial o primaria.

\section{De una visión de la hipertensión arterial basada en el riesgo hacia una basada en el estado fisiopatológico}

Sin duda uno de los puntos álgidos de discusión reciente ha sido la definición de la hipertensión arterial, y en los últimos años, los estudios realizados han llevado a proponer diferencias de hasta $10 \mathrm{mmHg}$ en el punto de corte entre las guías norteamericanas y europeas para establecer quien está hipertenso ${ }^{2,3}$. A pesar de las diferencias en los puntos de corte, ambas clasificaciones siguen utilizando básicamente el riesgo atribuible a la cifra de presión arterial como el criterio para hacer el diagnóstico y el tratamiento, es decir, la hipertensión es vista como una condición en la que se interviene el riesgo, y no como una condición nosológica con fisiopatología diversa y con características definidas en cada paciente. Si bien han ocurrido algunos avances, cuando se hace el diagnóstico de hipertensión esencial seguimos "tratando" las cifras de presión sin la identificación apropiada de lo que ocurre en el sistema cardiovascular de un paciente hipertenso. La definición de hipertensión aún es epidemiológica y no fisiopatológica. El diagnóstico basado en las cifras de presión arterial se realiza mediante mediciones en el consultorio o con métodos ambulatorios más preci$\operatorname{sos}^{4}$, y hemos aprendido que los valores de presión arterial frecuentemente no se correlacionan con los hallazgos cardiocirculatorios que presentan los pacientes que tienen cifras elevadas de presión arterial ${ }^{5}$. El número de alteraciones en los sistemas de control de la presión arterial es amplio y el grado de compromiso metabólico, neuroendocrino, renal, arterial y cardiaco es diferente entre individuos clasificados como hipertensos, o sea, con cifras de presión arterial promedio en el consultorio $\geq 140 / 90 \mathrm{mmHg}$.

Por muchos años ha persistido el concepto de denominar hipertenso esencial a quien tiene elevación de la presión arterial sin una causa secundaria. Aceptar que sea una hipertensión esencial no debe significar desconocer lo que ocurre en el sistema circulatorio del paciente $o$ asumir que todo se resume en un aumento

\section{Correspondencia:}

*Dagnóvar Aristizábal-Ocampo

E-mail: dagnovar@gmail.com

DOI: 10.24875/RCCAR.M21000075 licencia CC BY-NC-ND (http://creativecommons.org/licenses/by-nc-nd/4.0/).

Disponible en internet: 07-01-2022 Rev Colomb Cardiol. 2021;28(6):515-518 www.rccardiologia.com 
de la actividad de sistemas vasoconstrictores (p. ej., renina-angiotensina), que elevan la resistencia vascular y cuyo tratamiento está dirigido a neutralizarlos. Con los avances desde nuestra investigación ${ }^{6}$ y la de otros grupos ${ }^{7}$ se ha hecho más claro que se puede estar hipertenso en presencia de cambios fisiopatológicos muy diferentes entre pacientes. Existen, por tanto, diversos perfiles hemodinámicos en los pacientes hipertensos esenciales. Las características hemodinámicas del paciente joven difieren de las observadas en el paciente de edad media 0 en el adulto mayor ${ }^{8}, y$ también son diferentes las respuestas neuroendocrina y renal. Por ende, considerar que todos los pacientes hipertensos están bajo la misma carga de sustancias vasoconstrictoras o con los mismos cambios en el volumen vascular es un error conceptual que tiene consecuencias para el diagnóstico y el tratamiento.

La homogenización de la fisiopatología de la hipertensión esencial (p. ej., atribuyendo a todo hipertenso una resistencia vascular sistémica elevada), un hecho que ha sido común hasta ahora, debe ser reemplazada por una mejor clasificación fisiopatológica. Tomando en cuenta solo la cifra de presión arterial no sabemos lo que biológicamente está sucediendo en nuestro paciente. Para entenderlo es necesario mirar la hipertensión arterial como una condición clínica con un amplio espectro de estados hemodinámicos, cada uno comandado por diversos factores endógenos, entre los que alguno puede predominar, pero es necesario saber cuál o cuáles alteraciones subsisten en un determinado paciente. Mediante el análisis combinado del flujo sanguíneo arterial, el tipo de hipertensión predominante y la respuesta de los grandes y pequeños vasos arteriales, ya podemos dar un verdadero «apellido» a la hipertensión de cada paciente para dejar de llamarla esencial; por tanto, es necesario indicar si la hipertensión es de origen neurogénico o cardiogénico, por aumento del volumen vascular, por vasoconstricción o por rigidez arterial, etc. Es indudable que pueden existir mezclas en estas alteraciones, lo cual hace parte de la diversidad fisiopatológica de esta condición. Al obtener una explicación hemodinámica (y fisiopatológica) para la hipertensión arterial en cada paciente, la prescripción terapéutica será más específica y estará menos afectada por el ensayo-error ${ }^{9}$. En la hipertensión arterial, al igual que en otras patologías como la insuficiencia cardiaca ${ }^{10}$, el espectro de alteraciones circulatorias se viene precisando cada vez mejor y ya podemos indicar cuál es el fenotipo hipertensivo de un paciente. En particular, nuestra investigación nos ha permitido obtener cinco fenotipos hipertensivos discretos a partir del estudio de las variables hemodinámicas fundamentales en pacientes con presión arterial alta (Fig. 1).

\section{La importancia del fenotipo clínico en la era de la medicina de precisión}

En un reporte del año 2011, el concilio de investigación nacional de los Estados Unidos de América indicó que la medicina de precisión busca «adaptar el tratamiento médico a las características individuales de cada paciente (...) e incluye la habilidad para clasificar individuos en subpoblaciones que difieren en su susceptibilidad a una enfermedad particular o en su respuesta a un tratamiento específico ${ }^{11}$. Saber qué sucede cardiocirculatoriamente en cada paciente hipertenso nos acerca a definir un mejor esquema terapéutico, farmacológico o no farmacológico, «a la medida" del individuo. Este paso hacia una medicina de precisión es necesario en la hipertensión arterial. Hoy sabemos que los tratamientos tienen efectos diferenciales según el tipo de hipertensión observada. El auge del polypill puede verse como una solución transitoria, muy práctica para mejorar las coberturas y el riesgo de un problema tan frecuente y con bajas tasas de control en las poblaciones; sin embargo, necesitamos más precisión terapéutica acorde con las particularidades endógenas de cada paciente. La terapéutica guiada por los perfiles hemodinámicos ha mostrado su utilidad en los pacientes hipertensos ${ }^{12}$. Con los progresos que están emergiendo en los métodos de medición (p. ej., presión arterial continua) y con los análisis predictivos basados en la inteligencia artificial lograremos una clasificación cada vez más confiable del fenotipo clínico y de la respuesta terapéutica del paciente (esto es, medicina de precisión en la hipertensión arterial) ${ }^{9}$.

\section{Las nuevas tecnologías y el automonitoreo de la presión arterial por el paciente}

Con los avances notables en los dispositivos vestibles (p. ej., relojes inteligentes, etc.), que serán utilizados para medir la presión arterial en forma continua, lograremos más claridad sobre el comportamiento biológico de la presión arterial y los determinantes de su variabilidad, que nos indicarán las interacciones que tienen las actividades de la vida diaria con la cifra de presión arterial. Adicionalmente, la posibilidad de medir la presión arterial en forma continua y no intrusiva permitirá 


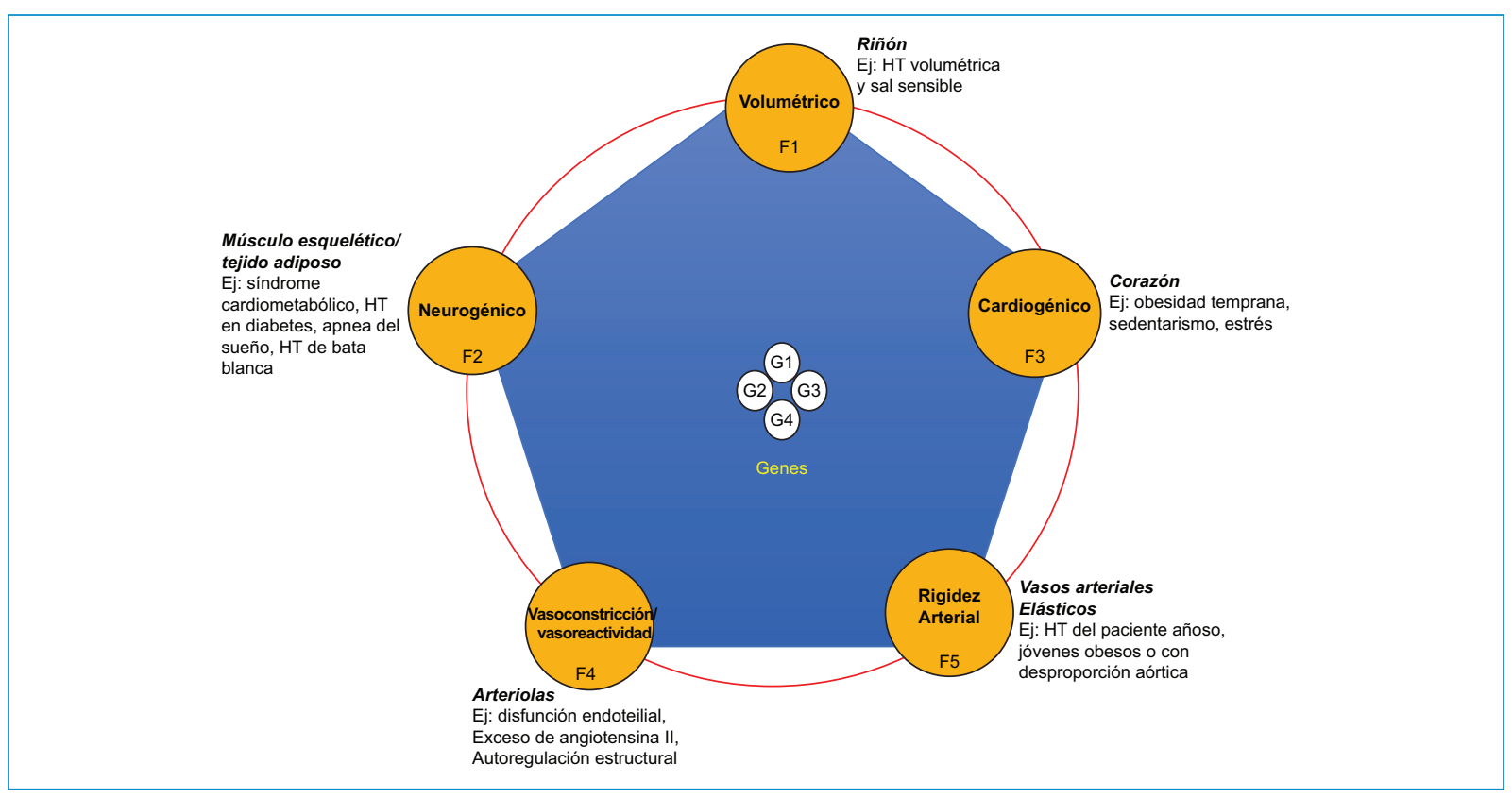

Figura 1. Fenotipos hemodinámicos hipertensivos. Se describen cinco fenotipos hemodinámicos discretos (F1 a F5) con su respectiva denominación (dentro del círculo) y los órganos que comandan la respuesta circulatoria de cada fenotipo en el texto (por fuera del círculo). También se dan ejemplos de formas clínicas que muestran las características de cada subgrupo hemodinámico. HT: hipertensión.

a los individuos entender por qué se elevan sus cifras de presión arterial e involucrarse en el (auto)cuidado de esta condición. Por otra parte, mediante el uso de diversos sensores será posible obtener no solo la presión arterial, sino también la frecuencia cardiaca, el electrocardiograma, mediciones de actividad física (con actigrafía), el estado emocional y el consumo de oxígeno, y correlacionar estos hallazgos con las cifras de presión arterial. Nos acercaremos cada vez más a descubrir por qué se presenta la hipertensión en un determinado individuo. Por último, el mayor conocimiento de los efectos biológicos de los cambios en los estilos de vida sobre las cifras de presión arterial modificará la frecuencia con la cual ciertos pacientes que antes se identificaban como hipertensos esenciales pasarán a tener formas de hipertensión no esenciales, tal como ha ocurrido en los pacientes hipertensos obesos con altos niveles de aldosterona y en otras formas nuevas de hipertensión, como las debidas al síndrome de apnea-hipopnea del sueño. Así, el porcentaje de pacientes hipertensos "esenciales» se reducirá notablemente.

En conclusión, en esta década comprenderemos mucho mejor las bases hemodinámicas y fisiopatológicas de la hipertensión en los humanos, y con ello avanzaremos a realizar un tratamiento eficaz "a la medida» del perfil hemodinámico de cada paciente, como se comienza a hacer hoy, por ejemplo, en la insuficiencia cardiaca $^{10}$. Preparémonos para estos grandes cambios que nos van a permitir guiar el tratamiento de nuestros pacientes utilizando la medicina de precisión, de acuerdo con el fenotipo hipertensivo.

\section{Financiamiento}

Este trabajo fue financiado por el Centro Clínico de Investigación SICOR, de Medellín, Colombia.

\section{Conflicto de intereses}

El autor no tiene conflictos de intereses.

\section{Bibliografía}

1. Aristizábal D. La hipertensión en el siglo XXI: los grandes cambios que nos esperan. Rev Colomb Cardiol. 2007;14:181-4.

2. Whelton PK, Carey RM, Aronow WS, Casey DE Jr., Collins KJ, Dennison Himmelfarb C, et al. 2017 ACC/AHA/AAPA/ABC/ACPM/AGS/ APhA/ASH/ASPC/NMA/PCNA Guideline for the prevention, detection, evaluation, and management of high blood pressure in adults: a report of the American College of Cardiology/American Heart Association Task Force on Clinical Practice Guidelines. J Am Coll Cardiol. 2018;71:e127-e248.

3. Williams B, Mancia G, Spiering W, Agabiti Rosei E, Azizi M, Burnier M, et al. 2018 ESC/ESH Guidelines for the management of arterial hypertension: The Task Force for the management of arterial hypertension of the European Society of Cardiology and the European Society of Hypertension. J Hypertens. 2018;36:1953-2041. 
Rev Colomb Cardiol. 2021;28(6)

4. Stergiou GS, Palatini P, Parati G, O'Brien E, Januszewicz A, Lurbe E, et al. 2021 European Society of Hypertension practice guidelines for office and out-of-office blood pressure measurement. J Hypertens. 2021;39:1293-302.

5. Cheang MH, Kowalik GT, Quail MA, Steeden JA, Hothi D, Tullus K, et al. The cardiovascular phenotype of childhood hypertension: a cardiac magnetic resonance study. Pediatr Radiol. 2019;49:727-36.

6. Álvarez-Montoya D, Madrid-Muñoz C, Escobar-Robledo L, Gallo-Villegas J, Aristizábal-Ocampo D. A novel method for the noninvasive estimation of cardiac output with brachial oscillometric blood pressure measurements through an assessment of arterial compliance. Blood Press Monit. 2021;26:426-36.

7. Krzesinski P, Gielerak GG, Kowal JJ. A "patient-tailored" treatment of hypertension with use of impedance cardiography: a randomized, prospective and controlled trial. Med Sci Monit. 2013;19:242-50.
8. Smith BE, Madigan VM. Understanding the haemodynamics of hypertension. Curr Hypertens Rep. 2018;20:29.

9. Savoia C, Volpe M, Grassi G, Borghi C, Agabiti Rosei E, Touyz RM Personalized medicine - a modern approach for the diagnosis and management of hypertension. Clin Sci (Lond). 2017;131:2671-85.

10. Rosano GMC, Moura B, Metra M, Böhm M, Bauersachs J, Ben Gal T, et al. Patient profiling in heart failure for tailoring medical therapy. A consensus document of the Heart Failure Association of the European Society of Cardiology. Eur J Heart Fail. 2021;23:872-81.

11. Toward Precision Medicine: Building a Knowledge Network for Biomedical Research and a New Taxonomy of Disease. The National Academies Collection: Reports funded by National Institutes of Health. Washington (DC); 2011.

12. Ferrario CM, Basile J, Bestermann W, Frohlich E, Houston M, Lackland DT, et al. The role of noninvasive hemodynamic monitoring in the evaluation and treatment of hypertension. Ther Adv Cardiovasc Dis. 2007;1:113-8. 